\title{
On submanifolds in locally symmetric spaces of noncompact type
}

\author{
JEAN-FRANÇOIS LAFONT \\ BENJAMIN SCHMIDT
}

\begin{abstract}
Given a connected, compact, totally geodesic submanifold $Y^{m}$ of noncompact type inside a compact locally symmetric space of noncompact type $X^{n}$, we provide a sufficient condition that ensures that $\left[Y^{m}\right] \neq 0 \in H_{m}\left(X^{n} ; \mathbb{R}\right)$; in low dimensions, our condition is also necessary. We provide conditions under which there exist a tangential map of pairs from a finite cover $(\bar{X}, \bar{Y})$ to the nonnegatively curved duals $\left(X_{u}, Y_{u}\right)$.
\end{abstract}

53C $35 ; 57 \mathrm{~T} 15,55 \mathrm{R} 37,57 \mathrm{R} 42,57 \mathrm{R} 45$

\section{Introduction}

In this paper, we propose to study totally geodesic submanifolds inside locally symmetric spaces. Let us start by fixing some notation: $\left(X^{n}, Y^{m}\right)$ will always refer to a pair of compact locally symmetric spaces of noncompact type, with $Y^{m} \subset X^{n}$ a totally geodesic submanifold. The spaces $X^{n}, Y^{m}$ will be locally modelled on $G / K, G^{\prime} / K^{\prime}$ respectively, where $G, G^{\prime}$ are a pair of semisimple Lie groups, and $K, K^{\prime}$ are a pair of maximal compact subgroups in the respective $G, G^{\prime}$. Note that, since $Y^{m} \subset X^{n}$ is totally geodesic, one can view $G^{\prime}$ as a subgroup of $G$, and hence one can take $K^{\prime}=K \cap G^{\prime}$. We will denote by $X_{u}=G_{u} / K, Y_{u}=G_{u}^{\prime} / K^{\prime}$ the nonnegatively curved dual symmetric spaces to the nonpositively curved spaces $G / K, G^{\prime} / K^{\prime}$.

Note that for a pair $\left(X^{n}, Y^{m}\right)$, the submanifold $Y^{m}$ is always homotopically nontrivial. Indeed, the inclusion induces a monomorphism on the level of fundamental groups. A more subtle question is whether the submanifold $Y^{m}$ is homologically nontrivial, ie whether $\left[Y^{m}\right] \neq 0 \in H_{m}\left(X^{n} ; \mathbb{R}\right)$ (or in $H_{m}\left(X^{n} ; \mathbb{Z}\right)$ ). Our first result provides a criterion for detecting when a totally geodesic submanifold $Y^{m}$ is homologically nontrivial (over $\mathbb{R}$ ) in $X^{n}$.

Theorem 1.1 Let $Y^{m} \hookrightarrow X^{n}$ be a compact totally geodesic submanifold of noncompact type inside a compact locally symmetric space of noncompact type, and denote by $\rho$ the map on cohomology $H^{m}\left(X_{u} ; \mathbb{R}\right) \rightarrow H^{m}\left(Y_{u} ; \mathbb{R}\right) \simeq \mathbb{R}$ induced by the embedding $Y_{u} \hookrightarrow X_{u}$. Then we have the following: 
- If $\left[Y^{m}\right]=0 \in H_{m}\left(X^{n} ; \mathbb{R}\right)$ then the map $\rho$ is identically zero.

- If $\rho$ is identically zero, and $m \leq m(\mathfrak{g})$, where $m(\mathfrak{g})$ is the Matsushima constant corresponding to the Lie algebra $\mathfrak{g}$ of the Lie group $G$, then we have that $\left[Y^{m}\right]=0 \in H_{m}\left(X^{n} ; \mathbb{R}\right)$.

Our proof of this first result is an adaptation of an argument of Matsushima [10] and relies on the existence of certain compatible maps (the Matsushima maps) from the real cohomology of the pair of nonnegatively curved duals $\left(X_{u}, Y_{u}\right)$ to the real cohomology of the nonpositively curved pair $\left(X^{n}, Y^{m}\right)$. It is reasonable to ask whether this map can be realized geometrically. Our second result, extending work of Okun [15], shows that this can sometimes be achieved rationally:

Theorem 1.2 Assume that $Y^{m} \hookrightarrow X^{n}$ is a totally geodesic embedding of compact, locally symmetric spaces of noncompact type. Furthermore, assume that the map $G_{u}^{\prime} \hookrightarrow G_{u}$ induced by the inclusion $Y \hookrightarrow X$ is a $\pi_{i}$-isomorphism, for $i<m$, and a surjection on $\pi_{m}$. Then there exists a finite cover $\bar{X}$ of $X^{n}$, and a connected lift $\bar{Y} \subset \bar{X}$ of $Y^{m}$, with the property that there exists a tangential map of pairs $(\bar{X}, \bar{Y}) \rightarrow\left(X_{u}, Y_{u}\right)$. If in addition we have $\operatorname{rk}\left(G_{u}\right)=\operatorname{rk}(K)$ and $\operatorname{rk}\left(G_{u}^{\prime}\right)=\operatorname{rk}\left(K^{\prime}\right)$, then the respective tangential maps induce the Matsushima maps on cohomology.

Since the tangent bundle of the submanifold $Y^{m}$ Whitney sum with the normal bundle of $Y^{m}$ in $X^{n}$ yields the restriction of the tangent bundle of $X^{n}$ to the submanifold $Y^{m}$, this gives the immediate:

Corollary 1.3 Under the hypotheses of Theorem 1.2, we have that the pullback of the normal bundle of $Y_{u}$ in $X_{u}$ is stably equivalent to the normal bundle of $\bar{Y}^{m}$ in $\bar{X}^{n}$.

In the previous corollary, we note that if $2 m+1 \leq n$, then these two bundles are in fact isomorphic (see for instance Husemoller [5, Chapter 8, Theorem 1.5]).

An example where the hypotheses of the Theorem 1.2 are satisfied arises in the situation where $Y^{m}, X^{n}$ are real hyperbolic manifolds. Specializing Corollary 1.3 to this situation, we obtain:

Corollary 1.4 Let $Y^{m} \hookrightarrow X^{n}$ be a totally geodesic embedding, where $X^{n}, Y^{m}$ are compact hyperbolic manifolds, and assume that $2 m+1 \leq n$. Then there exists a finite cover $\bar{X}$ of $X^{n}$, and a connected lift $\bar{Y}$ of $Y^{m}$, with the property that the normal bundle of $\bar{Y}$ in $\bar{X}$ is trivial. 
While the hypotheses of Theorem 1.2 are fairly technical, we point out that there exist several examples of inclusions $Y^{m} \hookrightarrow X^{n}$ satisfying the hypotheses of the theorem. The proof of Corollary 1.4, as well as a discussion of some further examples will be included at the end of Section 4. Finally, we will conclude the paper with various remarks and open questions in Section 5.

Acknowledgements This research was partially conducted during the period when B Schmidt was employed by the Clay Mathematics Institute as a Liftoff Fellow. The research of J-F Lafont was partly supported by the National Science Foundation under grant DMS - 0606002. The authors would like to thank the anonymous referee for pointing out a simplification in our original proof of Proposition 5.2.

\section{Background}

In this section, we provide some discussion of the statements of our theorems. We also introduce some of the ingredients that will be used in the proofs of our results.

\subsection{Dual symmetric spaces}

Let us start by recalling the definition of dual symmetric spaces:

Definition Given a symmetric space $G / K$ of noncompact type, we define the dual symmetric space in the following manner. Let $G_{\mathbb{C}}$ denote the complexification of the semisimple Lie group $G$, and let $G_{u}$ denote the maximal compact subgroup in $G_{\mathbb{C}}$. Since $K$ is compact, under the natural inclusions $K \subset G \subset G_{\mathbb{C}}$, we can assume that $K \subset G_{u}$ (up to conjugation). The symmetric space dual to $G / K$ is defined to be the symmetric space $G_{u} / K$. By abuse of language, if $X=\Gamma \backslash G / K$ is a locally symmetric space modelled on the symmetric space $G / K$, we will say that $X$ and $G_{u} / K$ are dual spaces.

Now assume that $Y^{m} \hookrightarrow X^{n}$ is a totally geodesic submanifold, where both $Y^{m}, X^{n}$ are locally symmetric spaces of noncompact type. Fixing a lift of $Y$, we have a totally geodesic embedding of the universal covers:

$$
G^{\prime} / K^{\prime}=\tilde{Y} \hookrightarrow \tilde{X}=G / K
$$

Corresponding to this totally geodesic embedding, we get a natural commutative diagram:

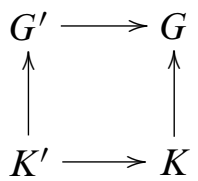

Algebraic ${ }^{8} \mathcal{G}$ Geometric Topology, Volume 6 (2006) 
which, after passing to the complexification, and descending to the maximal compacts, yields a commutative diagram:

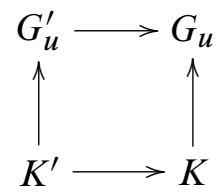

In particular, corresponding to the totally geodesic embedding $Y \hookrightarrow X$, we see that there is a totally geodesic embedding of the dual symmetric spaces $G_{u}^{\prime} / K^{\prime} \hookrightarrow G / K$.

\subsection{Classifying spaces}

For $G$ a continuous group let $E G$ denote a contractible space which supports a free $G$-action. The quotient space, denoted $B G$, is called a classifying space for principal $G$-bundles. This terminology is justified by the fact that, for any topological space $X$, there is a bijective correspondence between (1) isomorphism classes of principal $G$-bundles over $X$, and (2) homotopy classes of maps from $X$ to $B G$. Note that the spaces $E G$ are only defined up to $G$-equivariant homotopies, and likewise the spaces $B G$ are only defined up to homotopy. Milnor [11] gave a specific construction, for a Lie group $G$, of a space homotopy equivalent to $B G$. The basic fact we will require concerning classifying spaces is the following:

Theorem 2.1 If $H$ is a closed subgroup of the Lie group $G$, then there exists a natural map $B H \rightarrow B G$ between the models constructed by Milnor; furthermore this map is a fiber bundle with fiber the homogenous space $G / H$.

\subsection{Okun's construction}

Okun established [15, Theorem 5.1] the following nice result:

Theorem 2.2 Let $X=\Gamma \backslash G / K$ and $X_{u}=G_{u} / K$ be dual symmetric spaces. Then there exists a finite sheeted cover $\bar{X}$ of $X$ (ie a subgroup $\bar{\Gamma}$ of finite index in $\Gamma$, $\bar{X}=\bar{\Gamma} \backslash G / K)$, and a tangential map $k: \bar{X} \rightarrow X_{u}$.

This was subsequently used by Okun to exhibit exotic smooth structures on certain compact locally symmetric spaces of noncompact type [16], and by Aravinda-Farrell in their construction of exotic smooth structures on certain quaternionic hyperbolic manifolds supporting metrics of strict negative curvature [1]. More recently, this was used by Lafont-Roy [6] to give an elementary proof of the Hirzebruch proportionality 
principle for Pontrjagin numbers, as well as (non)vanishing results for Pontrjagin numbers of the Gromov-Thurston examples of manifolds with negative sectional curvature.

Since it will be relevant to our proof of the main theorem, we briefly recall the construction of the finite cover that appears in Okun's argument for Theorem 2.2. Starting from the canonical principle fiber bundle

$$
\Gamma \backslash G \rightarrow \Gamma \backslash G / K=X
$$

with structure group $K$ over the base space $X$, we can extend the structure group to the group $G$, yielding the flat principle bundle:

$$
\Gamma \backslash G \times_{K} G=G / K \times_{\Gamma} G \longrightarrow \Gamma \backslash G / K=X
$$

Further extending the structure group to $G_{\mathbb{C}}$ yields a flat bundle with a complex linear algebraic structure group. A result of Deligne and Sullivan [3] implies that there is a finite cover $\bar{X}$ of $X$ where the pullback bundle is trivial; since $G_{u}$ is the maximal compact in $G_{\mathbb{C}}$, the bundle obtained by extending the structure group from $K$ to $G_{u}$ is trivial as well. In terms of the classifying spaces, this yields the commutative diagram:

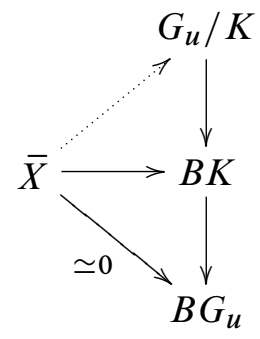

Upon homotoping the bottom diagonal map to a point, one obtains that the image of the horizontal map lies in the fiber above a point, ie inside $G_{u} / K$, yielding the dotted diagonal map in the above diagram. Okun then proceeds to show that the map to the fiber is the desired tangential map (since the pair of maps to $B K$ classify the respective canonical $K$-bundles on $\bar{X}$ and $G_{u} / K$, and the canonical $K$-bundles determine the respective tangent bundles).

\subsection{Matsushima's map}

Matsushima [10] constructed a map on cohomology $j^{*}: H^{*}\left(G_{u} / K ; \mathbb{R}\right) \rightarrow H^{*}(X ; \mathbb{R})$ whenever $X$ is a compact locally symmetric space modelled on $G / K$. We will require the following fact concerning the Matsushima map:

Algebraic ${ }^{6} \mathcal{G}$ Geometric Topology, Volume 6 (2006) 
Theorem 2.3 (Matsushima [10]) The map $j^{*}$ is always injective. Furthermore, there exists a constant $m(\mathfrak{g})$ (called the Matsushima constant) depending solely on the Lie algebra $\mathfrak{g}$ of the Lie group $G$, with the property that the Matushima map $j^{*}$ is a surjection in cohomology up to the dimension $m(\mathfrak{g})$.

The specific value of the Matsushima constant for the locally symmetric spaces that are Kähler can be found in [10]. We also point out the following result of Okun [15, Theorem 6.4]:

Theorem 2.4 Let $X=\Gamma \backslash G / K$ be a compact locally symmetric space, and $\bar{X}$, $t: \bar{X} \rightarrow G_{u} / K$ the finite cover and tangential map constructed in Theorem 2.2. If the groups $G_{u}$ and $K$ have equal rank, then the induced map $t^{*}$ on cohomology coincides with Matsushima's map $j^{*}$.

\section{Detecting homologically essential submanifolds}

In this section, we provide a proof of Theorem 1.1, which gives a criterion for establishing when a totally geodesic submanifold $Y \subset X$ in a locally symmetric space of noncompact type, is homologically nontrivial.

Proof of Theorem 1.1 In order to establish the theorem, we make use of differential forms. If a group $H$ acts on a smooth manifold $M$, we let $\Omega^{H}(M)$ denote the complex of $H$-invariant differential forms on $M$. Let $X=\Gamma \backslash G / K, Y=\Lambda \backslash G^{\prime} / K^{\prime}$ be the pair of compact locally symmetric spaces, and $X_{u}=G_{u} / K, Y_{u}=G_{u}^{\prime} / K^{\prime}$ be the corresponding dual spaces. We now consider the following four complexes of differential forms: (1) $\Omega^{G}(G / K),(2) \Omega^{G^{\prime}}\left(G^{\prime} / K^{\prime}\right)$, (3) $\Omega^{\Gamma}(G / K)$ and (4) $\Omega^{\Lambda}\left(G^{\prime} / K^{\prime}\right)$.

We now observe that the cohomology of the first two complexes can be identified with the cohomology of $X_{u}, Y_{u}$ respectively. Indeed, we have the sequence of natural identifications:

$$
\Omega^{G}(G / K)=H^{*}(\mathfrak{g}, \mathfrak{t})=H^{*}\left(\mathfrak{g}_{u}, \mathfrak{t}\right)=\Omega^{G_{u}}\left(G_{u} / K\right)
$$

The first and third equalities come from the identification of the complex of harmonic forms with the relative Lie algebra cohomology. The second equality comes via the dual Cartan decompositions: $\mathfrak{g}=\mathfrak{t} \oplus \mathfrak{p}$ and $\mathfrak{g}_{u}=\mathfrak{t} \oplus i \mathfrak{p}$. Since $X_{u}=G_{u} / K$ is a compact closed manifold, and $\Omega^{G_{u}}\left(G_{u} / K\right)$ is the complex of harmonic forms on $X_{u}$, Hodge theory tells us that the cohomology of the complex $\Omega^{G_{u}}\left(G_{u} / K\right)$ is just the cohomology of $X_{u}$. The corresponding analysis holds for $\Omega^{G^{\prime}}\left(G^{\prime} / K^{\prime}\right)$. 
Next we note that the cohomology of the last two complexes can be identified with the cohomology of $X, Y$ respectively. This just comes from the fact that the projection $G / K \rightarrow \Gamma \backslash G / K=X$ induces the isomorphism of complexes $\Omega^{\Gamma}(G / K)=\Omega(X)$, and similarly for $Y$.

Now observe that the four complexes fit into a commutative diagram of chain complexes:

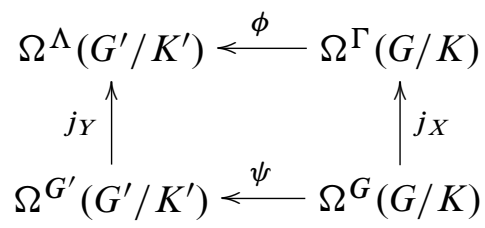

Let us briefly comment on the maps in the diagram. The vertical maps are obtained from the fact that $\Gamma \leq G$, so that any $G$-invariant form can be viewed as a $\Gamma$-invariant form, and similarly for $\Lambda \leq G^{\prime}$.

For the horizontal maps, one observes that $G^{\prime} / K^{\prime} \hookrightarrow G / K$ is an embedding, hence any form on $G / K$ restricts to a form on $G^{\prime} / K^{\prime}$. We also have the inclusion $\Lambda \leq \Gamma$, and hence the restriction of a $\Gamma$-invariant form on $G / K$ yields a $\Lambda$-invariant form on $G^{\prime} / K^{\prime}$. This is the horizontal map in the top row. One obtains the horizontal map in the bottom row similarly.

Now passing to the homology of the chain complexes, and using the identifications discussed above, we obtain a commutative diagram in dimension $m=\operatorname{dim}(Y)=$ $\operatorname{dim}\left(G_{u}^{\prime} / K^{\prime}\right)$ :

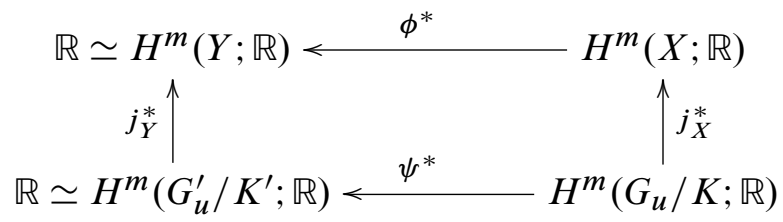

Note that the two vertical maps defined here are precisely the Matsushima maps for the respective locally symmetric spaces. Since Matsushima's map is always injective, and the cohomology of $H^{m}\left(G_{u}^{\prime} / K^{\prime} ; \mathbb{R}\right)$ and $H^{m}(Y ; \mathbb{R})$ are both one-dimensional, we obtain that $j_{Y}^{*}$ is an isomorphism. Likewise $j_{X}^{*}$ is always injective, and if $m \leq m(\mathfrak{g})$ then $j_{X}^{*}$ is also surjective (and hence $j_{X}^{*}$ is an isomorphism as well). This implies the following two facts:

- If $\phi^{*}$ is identically zero, then $\psi^{*}$ is identically zero.

- If furthermore $m \leq m(\mathfrak{g})$, then both vertical maps are isomorphisms, and we have that $\psi^{*}$ is identically zero if and only if $\phi^{*}$ is identically zero. 
Now observe that both of the horizontal maps coincide with the maps induced on cohomology by the respective inclusions $Y \hookrightarrow X$ and $G_{u}^{\prime} / K^{\prime} \hookrightarrow G_{u} / K$; indeed the maps are obtained by restricting the forms defined on the ambient manifold to the appropriate submanifold. In particular, the map $\psi^{*}$ coincides with the map $\rho$ that appears in the statement of our theorem. On the other hand, from the Kronecker pairing, the map $\phi^{*}$ is nonzero precisely when $\left[Y^{m}\right] \neq 0 \in H_{m}(X ; \mathbb{R})$. Combining these observations with the two facts in the previous paragraph completes the proof of Theorem 1.1.

Remark (1) The Matsushima map is only defined on the real cohomology (since it passes through differential forms), and as a result, cannot be used to obtain any information on torsion elements in $H^{k}\left(X^{n} ; \mathbb{Z}\right)$.

(2) We remark that the proof of Theorem 1.1 applies equally well to lower-dimensional cohomology (using the fact that Matsushima's map is injective in all dimensions), and gives the following lower-dimensional criterion. Assume that the map $H^{k}\left(X_{u}^{n} ; \mathbb{R}\right) \rightarrow$ $H^{k}\left(Y_{u}^{m} ; \mathbb{R}\right)$ has image containing a nonzero class $\alpha$, and let $i(\alpha) \in H^{k}\left(Y^{m} ; \mathbb{R}\right)$ be the nonzero image class under the Matsushima map. Then the homology class $\beta \in H_{k}\left(Y^{m} ; \mathbb{R}\right)$ dual (under the Kronecker pairing) to $i(\alpha)$ has nonzero image in $H_{k}\left(X^{n} ; \mathbb{R}\right)$ under the map induced by the inclusion $Y^{m} \hookrightarrow X^{n}$.

\section{Pairs of tangential maps}

In this section, we proceed to give a proof of Theorem 1.2, establishing the existence of pairs of tangential maps from the pair $(\bar{X}, \bar{Y})$ to the pair $\left(X_{u}, Y_{u}\right)$.

Proof of Theorem 1.2 We start out by applying Theorem 2.2, which gives us a finite cover $\bar{X}$ of $X$ with the property that the natural composite map $\bar{X} \rightarrow B K \rightarrow B G_{u}$ is homotopic to a point. Note that this map classifies the principle $G_{u}$ bundle over $\bar{X}$.

Now let $\bar{Y} \hookrightarrow \bar{X}$ be a connected lift of the totally geodesic subspace $Y \hookrightarrow X$. Observe that, by naturality, we have a commutative diagram:

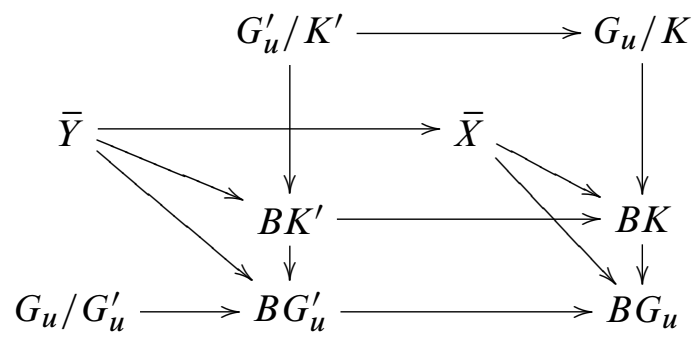

Algebraic 83 Geometric Topology, Volume 6 (2006) 
By Okun's result, the composite map $\bar{X} \rightarrow B G_{u}$ is homotopic to a point via a homotopy $H: \bar{X} \times I \rightarrow B G_{u}$. We would like to establish the existence of a homotopy $F: \bar{Y} \times I \rightarrow$ $B G_{u}^{\prime}$ with the property that the following diagram commutes:

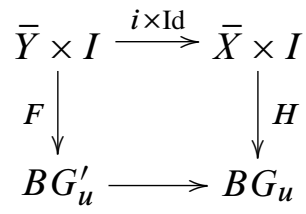

Indeed, if we had the existence of such a compatible pair of homotopies, then one can easily complete the argument: since each of the vertical columns in the diagram are fiber bundles, we see that after applying the pair of compatible homotopies, the images of $(\bar{X}, \bar{Y})$ lies in the pair of fibers $\left(G_{u} / K, G_{u}^{\prime} / K^{\prime}\right)$. This yields a pair of compatible lifts, yielding a commutative diagram of the form:

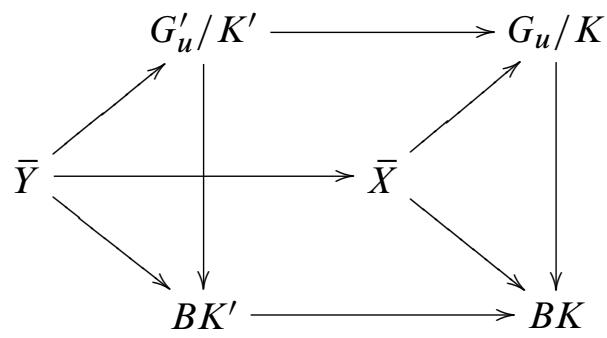

Since the pair of maps to $B K^{\prime}$ (respectively $B K$ ) classify the canonical $K^{\prime}$-bundle structures on $\bar{Y}, G_{u}^{\prime} / K^{\prime}$ (respectively the canonical $K$-bundle structure on $\bar{X}, G_{u} / K$ ), and since these bundles canonically determine the tangent bundles of these spaces $[15$, Lemma 2.3], commutativity of the diagram immediately gives us tangentiality of the maps $\bar{Y} \rightarrow G_{u}^{\prime} / K^{\prime}$ (respectively, of the map $\bar{X} \rightarrow G_{u} / K$ ).

In order to show the existence of the compatible homotopy $F: \bar{Y} \times I \rightarrow B G_{u}^{\prime}$, we start by observing that the bottom row of the commutative diagram is in fact a fibration

$$
G_{u} / G_{u}^{\prime} \rightarrow B G_{u}^{\prime} \rightarrow B G_{u} .
$$

Since $\bar{Y}$ is embedded in $\bar{X}$, we see that the homotopy $H$ induces by restriction a homotopy $H: \bar{Y} \times I \rightarrow B G_{u}$. Since the bottom row is a fibration, we may lift this homotopy to a homotopy $\widetilde{H}: \bar{Y} \times I \rightarrow B G_{u}^{\prime}$, with the property that $\widetilde{H}_{0}$ coincides with the map $\bar{Y} \rightarrow B G_{u}^{\prime}$ which classifies the canonical principle $G_{u}^{\prime}$ bundle over $\bar{Y}$. Unfortunately, we do not know, a priori, that the time one map $\tilde{H}_{1}$ maps $\bar{Y}$ to a point in $B G_{u}^{\prime}$. Indeed, we merely know that $\widetilde{H}_{1}(\bar{Y})$ lies in the preimage of a point in $B G_{u}$, ie in the fiber $G_{u} / G_{u}^{\prime}$. Our next goal is to establish that the map $\widetilde{H}_{1}: \bar{Y} \rightarrow G_{u} / G_{u}^{\prime}$ is nullhomotopic. If this were the case, we could concatenate the homotopy $\widetilde{H}$ taking $\bar{Y}$ 
into the fiber $G_{u} / G_{u}^{\prime}$ with a homotopy contracting $\widetilde{H}_{1}: \bar{Y} \rightarrow G_{u} / G_{u}^{\prime}$ to a point within the fiber. This would yield the desired homotopy $F$.

In order to establish that $\widetilde{H}_{1}: \bar{Y} \rightarrow G_{u} / G_{u}^{\prime}$ is nullhomotopic, we merely note that we have the fibration

$$
G_{u}^{\prime} \rightarrow G_{u} \rightarrow G_{u} / G_{u}^{\prime}
$$

From the corresponding long exact sequence in homotopy groups, and using the fact that the inclusion $G_{u}^{\prime} \hookrightarrow G_{u}$ induces a $\pi_{i}$-isomorphism for $i<m$ and a surjection on $\pi_{m}$, we immediately obtain that $\pi_{i}\left(G_{u} / G_{u}^{\prime}\right) \cong 0$ for $i \leq m$. Since the dimension of the manifold $\bar{Y}$ is $m$, we can now conclude that the map $\widetilde{H}_{1}$ is nullhomotopic. Indeed, taking a cellular decomposition of $\bar{Y}$ with a single 0 -cell, one can recursively contract the image of the $i$-skeleton to the image of the 0 -cell: the obstruction to doing so lies in $\pi_{i}\left(G_{u} / G_{u}^{\prime}\right)$, which we know vanishes. This yields that $\tilde{H}_{1}$ is nullhomotopic, which by our earlier discussion, implies the existence of a tangential map of pairs $(\bar{X}, \bar{Y}) \rightarrow\left(X_{u}, Y_{u}\right)$. Finally, to conclude we merely point out the Okun has shown (see Theorem 2.4) that in the case where the rank of $G_{u}$ equals the rank of $K$, the tangential map he constructed induces the Matsushima map on cohomology. Our construction restricts to Okun's construction on both $X$ and $Y$, and from the hypothesis on the ranks, so we conclude that the tangential map of pairs induces the Matsushima map on the cohomology of each of the two spaces. This concludes the proof of Theorem 1.2 .

Remark We observe that the argument given above, for the case of a pair $\left(X^{n}, Y^{m}\right)$, can readily be adapted to deal with any descending chain of totally geodesic submanifolds. More precisely, assume that we have a series of totally geodesic embeddings $X^{n}=Y_{k} \supset \cdots \supset Y_{2} \supset Y_{1}$, with the property that each $Y_{j}$ is a closed locally symmetric space of noncompact type. Further assume that, if $\left(Y_{j}\right)_{u}=\left(G_{j}\right)_{u} / K_{j}$ denotes the compact duals, the maps $\left(G_{j}\right)_{u} \hookrightarrow\left(G_{j+1}\right)_{u}$ induced by the inclusions $Y_{j} \hookrightarrow Y_{j+1}$ are $\pi_{i}$ isomorphisms for $i<\operatorname{dim}\left(Y_{j}\right)$ and a surjection on $\pi_{i}\left(i=\operatorname{dim}\left(Y_{j}\right)\right)$. Then there exists a finite cover $\bar{X}^{n}=\bar{Y}_{k}$ of $X^{n}$, and connected lifts $\bar{Y}_{j}$ of $Y_{j}$, having the property that:

- we have containments $\bar{Y}_{j} \subset \bar{Y}_{j+1}$, and

- there exists a map $\left(\bar{Y}_{k}, \ldots, \bar{Y}_{1}\right) \rightarrow\left(\left(Y_{k}\right)_{u}, \ldots,\left(Y_{1}\right)_{u}\right)$ which restricts to a tangential map from each $\bar{Y}_{j}$ to the corresponding $\left(Y_{j}\right)_{u}$.

This is shown by induction on the length of a descending chain. We leave the details to the interested reader. 
We now proceed to show Corollary 1.4, that is to say, that in the case where $X^{n}$ is real hyperbolic, and $Y^{m} \hookrightarrow X^{n}$ is totally geodesic, there exists a finite cover $\bar{X}$ of $X^{n}$ and a connected lift $\bar{Y}$ of $Y^{m}$, with the property that the normal bundle of $\bar{Y}$ in $\bar{X}$ is trivial.

Proof of Corollary 1.4 We first observe that, provided one could verify the hypotheses of Theorem 1.2 for the pair $\left(X^{n}, Y^{m}\right)$, the corollary would immediately follow. Note that in this case, the dual spaces $X_{u}$ and $Y_{u}$ are spheres of dimension $n$ and $m$ respectively. This implies that the totally geodesic embedding $Y_{u} \hookrightarrow X_{u}$ is in fact a totally geodesic embedding $S^{m} \hookrightarrow S^{n}$, forcing the normal bundle to $Y_{u}$ in $X_{u}$ to be trivial. But now Corollary 1.3 to the Theorem 1.2 immediately yields Corollary 1.4.

So we are left with establishing the hypotheses of Theorem 1.2 for the pair $\left(X^{n}, Y^{m}\right)$. We observe that in this situation we have the groups $G_{u} \cong S O(n+1)$, and $G_{u}^{\prime} \cong$ $S O(m+1)$. Furthermore, there is essentially a unique totally geodesic embedding $S^{m} \hookrightarrow S^{n}$, hence we may assume that the embedding $G_{u}^{\prime} \hookrightarrow G_{u}$ is the canonical one. But now we have the classical facts that (1) the embeddings $S O(m+1) \hookrightarrow S O(n+1)$ induce isomorphisms on $\pi_{i}$ for $i<m$ and (2) that the embedding induces a surjection $\pi_{m}(S O(m+1)) \rightarrow \pi_{m}(S O(n+1))$. Indeed, this is precisely the range of dimensions where the homotopy groups stabilize [12]. This completes the verification of the hypotheses, and hence the proof of Corollary 1.4.

We now proceed to give an example of an inclusion $Y^{m} \hookrightarrow X^{n}$ satisfying the hypotheses of our theorem. Our spaces will be modelled on complex hyperbolic spaces, namely we have:

$$
\begin{aligned}
Y^{2 m} & =\Lambda \backslash \mathbb{C} \mathbb{M}^{m}=\Lambda \backslash S U(m, 1) / S(U(m) \times U(1)) \\
X^{2 n} & =\Gamma \backslash \mathbb{C} \mathbb{G}^{n}=\Gamma \backslash S U(n, 1) / S(U(n) \times U(1))
\end{aligned}
$$

To construct such pairs, one starts with the standard inclusion of $S U(m, 1) \hookrightarrow S U(n, 1)$, which induces a totally geodesic embedding $\mathbb{C}^{m} \hookrightarrow \mathbb{C} \mathbb{Q}^{n}$. One can now construct explicitly (by arguments similar to those in Gromov and Piatetski-Shapiro [4]) an arithmetic uniform lattice $\Lambda \leq S U(m, 1)$ having an extension to an arithmetic uniform lattice $\Gamma \leq S U(n, 1)$. Quotienting out by these lattices gives the desired pair.

Let us now consider these examples in view of our Theorem 1.2. First of all, we have that the respective complexifications are $G_{\mathbb{C}}^{\prime}=S L(m+1, \mathbb{C})$ and $G_{\mathbb{C}}=S L(n+1, \mathbb{C})$, with the natural embedding

$$
G_{\mathbb{C}}^{\prime}=S L(m+1, \mathbb{C}) \hookrightarrow S L(n+1, \mathbb{C})=G_{\mathbb{C}} .
$$


Looking at the respective maximal compacts, we see that $G_{u}^{\prime}=S U(m+1), G_{u}=$ $S U(n+1)$, and the inclusion is again the natural embeddings

$$
G_{u}^{\prime}=S U(m+1) \hookrightarrow S U(n+1)=G_{u} .
$$

Hence the homotopy condition in our theorem boils down to asking whether the natural embedding $S U(m+1) \hookrightarrow S U(n+1)$ induces isomorphisms on the homotopy groups $\pi_{i}$, where $i \leq \operatorname{dim}\left(Y^{2 m}\right)=2 m$. But it is a classical fact that the natural embedding induces isomorphisms in all dimensions $i<2(m+1)=2 m+2$, since this falls within the stable range for the homotopy groups (and indeed, one could use complex Bott periodicity to compute the exact value of these homotopy groups [12]). Finally, we observe that in this context, the dual spaces are complex projective spaces, and the embedding of dual spaces is the standard embedding $\mathbb{C} P^{m} \hookrightarrow \mathbb{C} P^{n}$. It is well known that for the standard embedding, we have that the induced map on cohomology $H^{*}\left(\mathbb{C} P^{n}\right) \rightarrow H^{*}\left(\mathbb{C} P^{m}\right)$ is surjective on cohomology. Our Theorem 1.1 now tells us that $Y^{2 m} \hookrightarrow X^{2 n}$ is homologically nontrivial. Furthermore, we note that for these manifolds, $\operatorname{rk}\left(G_{u}\right)=\operatorname{rk}(K)$ and $\operatorname{rk}\left(G_{u}^{\prime}\right)=\operatorname{rk}\left(K^{\prime}\right)$, and hence Theorem 1.2 tells us that the cohomological map from the proof of Theorem 1.1 can be (rationally) realized via a tangential map of pairs.

\section{Concluding remarks}

We conclude this paper with a few comments and questions. First of all, in view of our Theorem 1.1, it is reasonable to ask for the converse:

Question Given an element $\alpha \in H_{m}\left(X^{n} ; \mathbb{R}\right)$, is there an $m$-dimensional totally geodesic submanifold $Y^{m}$ with $\left[Y^{m}\right]=\alpha$ ?

A cautionary example for the previous question is provided by the following:

Proposition 5.1 Let $X$ be a compact hyperbolic 3-manifold that fibers over $S^{1}$, with fiber a surface $F$ of genus $\geq 2$. Then the homology class represented by $[F] \in H_{2}(X ; \mathbb{Z})$ cannot be represented by a totally geodesic submanifold.

Proof Assume that there were such a totally geodesic submanifold $Y \subset X$, and observe that since $Y$ is totally geodesic, we have an embedding $\pi_{1}(Y) \hookrightarrow \pi_{1}(X)$. Furthermore, since $X$ fibers over $S^{1}$ with fiber $F$, we also have a short exact sequence:

$$
0 \rightarrow \pi_{1}(F) \rightarrow \pi_{1}(X) \rightarrow \mathbb{Z} \rightarrow 0
$$


Our goal is to show that $\pi_{1}(Y) \subset \pi_{1}(F)$. Indeed, if we could establish this containment, one could then argue as follows: since $Y$ is a compact surface, covering space theory implies that $\pi_{1}(Y) \subset \pi_{1}(F)$ is a finite index subgroup. Now pick a point $x$ in the universal cover $\tilde{X} \cong \mathbb{H}^{3}$, and consider the subset $\Lambda_{Y} \subset \partial^{\infty} \mathbb{H}^{3}=S^{2}$ obtained by taking the closure of the $\pi_{1}(Y)$-orbit of $x$. Since $Y$ is assumed to be totally geodesic, the subset $\Lambda \subset S^{2}$ is a tamely embedded $S^{1}$ (identified with the boundary at infinity of a suitably chosen totally geodesic lift $\tilde{Y} \cong \mathbb{W}^{2}$ of $Y$ ). On the other hand, since $\pi_{1}(Y)$ has finite index in $\pi_{1}(F)$, we have that $\Lambda_{Y}$ must coincide with $\Lambda_{F}$, the closure of the $\pi_{1}(F)$-orbit of $x$. But the latter, by a well-known result of Cannon-Thurston is known to be the entire boundary at infinity (see for instance Mitra [14]).

So we are left with establishing that $\pi_{1}(Y) \subset \pi_{1}(F)$. In order to see this, let us consider the cohomology class $\alpha_{F} \in H^{1}(X ; \mathbb{Z})$ which is Poincare dual to the class $[F] \in H_{2}(X ; \mathbb{Z})$. Now recall that the evaluation of the cohomology class $\alpha_{F}$ on an element $[\gamma] \in H_{1}(X ; \mathbb{Z})$ can be interpreted geometrically as the intersection number of the representing curve $\gamma$ with the surface $F$. Furthermore, we have that the group $H_{1}(X ; \mathbb{Z})$ is generated by the image of $H_{1}(F ; \mathbb{Z})$, under the inclusion $F \hookrightarrow X$, along with an element $[\eta] \in H_{1}(X ; \mathbb{Z})$ mapping to $\left[S^{1}\right] \in H_{1}\left(S^{1} ; \mathbb{Z}\right)$. Here $\eta$ is chosen to be a closed loop in $M$ with the property that $\eta$ maps homeomorphically to the base $S^{1}$ (preserving orientations) under the projection map. This gives us the following two facts:

- The class $\alpha_{F}$ evaluates to 1 on the element $[\eta]$, since $F \cap \eta$ is a single transverse point.

- The class $\alpha_{F}$ evaluates to zero on the image of $H_{1}(F ; \mathbb{Z})$ in the group $H_{1}(X ; \mathbb{Z})$. This follows from the fact that the surface $F$ has trivial normal bundle in $X$, allowing any curve in $F$ representing (the image of) an element in $H_{1}(F ; \mathbb{Z})$ to be homotoped to a curve disjoint from $F$.

Furthermore, since we are assuming that $[Y]=[F] \in H_{2}(X ; \mathbb{Z})$, we know that we have an identification of Poincaré duals $\alpha_{Y}=\alpha_{F}$.

Now let us assume that $\pi_{1}(Y)$ is not contained in $\pi_{1}(F)$, and observe that this implies that there exists a closed loop $\gamma \subset Y$ having the property that under the composition $Y \hookrightarrow X \rightarrow S^{1}$, the class $[\gamma] \in H_{1}(Y ; \mathbb{Z})$ maps to $k \cdot\left[S^{1}\right] \in H_{1}\left(S^{1} ; \mathbb{Z}\right)$ (and $\left.k \neq 0\right)$. We now proceed to compute, in two different ways, the evaluation of the cohomology classes $\alpha_{Y}=\alpha_{F}$ on a suitable multiple of the homology class $[\gamma]$.

Firstly, from the comments above, we can write $[\gamma]$ as the sum of $k \cdot[\eta]$, along with an element $\beta$, where $\beta$ lies in the image of $H_{1}(F ; \mathbb{Z})$. By linearity of the Kronecker 
pairing, along with the two facts from the previous paragraph, we obtain:

$$
\alpha_{F}([\gamma])=\alpha_{F}(\beta)+k \alpha_{F}([\eta])=k \neq 0
$$

Secondly, observe that $Y$ is assumed to be embedded in $X$, and represents the nonzero homology class $[Y]=[F] \in H_{2}(X ; Z)$. This implies that $Y$ must be orientable, and hence has trivial normal bundle in $X$. In particular, the curve $\gamma \subset Y$ can be homotoped (within $X$ ) to have image disjoint from $Y$. Since the integer $\alpha_{Y}([\gamma])$ can be computed geometrically as the intersection number of the curve $\gamma$ with $Y$, we conclude that $\alpha_{Y}([\gamma])=0$.

Combining the two observations above, we see that $0=\alpha_{Y}([\gamma])=\alpha_{F}([\gamma]) \neq 0$, giving us the desired contradiction. This completes the proof of the proposition.

We observe that Thom [17] has shown that in dimensions $0 \leq k \leq 6$ and $n-2 \leq k \leq n$, every integral homology class can be represented by an immersed submanifold. In general however, there can exist homology classes which are not representable by submanifolds (see for instance Bohr, Hanke and Kotschick [2]). The question above asks for a more stringent condition, namely that the immersed submanifold in question be totally geodesic. We believe that the weaker question is also of some interest, namely:

Question Find an example $X^{n}$ of a compact locally symmetric space of noncompact type, and a homology class in some $H_{k}\left(X^{n} ; \mathbb{Z}\right)$ which cannot be represented by an immersed submanifold.

Now our original motivation for looking at totally geodesic submanifolds inside locally symmetric spaces was the desire to exhibit lower-dimensional bounded cohomology classes. In [7], the authors showed that for the fundamental group $\Gamma$ of a compact locally symmetric space of noncompact type $X^{n}$, the comparison map from bounded cohomology to ordinary cohomology:

$$
H_{b}^{*}(\Gamma) \rightarrow H^{*}(\Gamma)
$$

is surjective in dimension $n$. The proof actually passed through the dual formulation, and showed that the $L^{1}$ (pseudo)-norm of the fundamental class $\left[X^{n}\right] \in H_{n}\left(X^{n} ; \mathbb{R}\right.$ ) is nonzero. Now given a totally geodesic embedding $Y \hookrightarrow X$ of the type considered in this paper, it is tempting to guess that the homology class $[Y]$ also has nonzero $L^{1}$ (pseudo)-norm. Of course, this naive guess fails, since one can find examples where $[Y]=0 \in H_{m}\left(X^{n} ; \mathbb{R}\right)$. The problem is that despite the fact that the intrinsic $L^{1}$ (pseudo)-norm of $[Y]$ is nonzero, the extrinsic $L^{1}$ (pseudo)-norm of $[Y]$ is zero. In 
other words, one can represent the fundamental class of $Y$ more efficiently by using simplices that actually do not lie in $Y$ (despite the fact that $Y$ is totally geodesic). The authors were unable to answer the following:

Question Assume that $Y$ and $X$ are compact locally symmetric spaces of noncompact type, that $Y \subset X$ is a totally geodesic embedding, and that $Y$ is orientable with $[Y] \neq 0 \in H_{m}\left(X^{n} ; \mathbb{R}\right)$. Does it follow that the dual cohomology class $\beta \in H^{m}\left(X^{n} ; \mathbb{R}\right)$ (via the Kronecker pairing) has a bounded representative?

Now one situation in which nonvanishing of the $L^{1}$ (pseudo)-norm would be preserved is the case where $Y \hookrightarrow X$ is actually a retract of $X$. Hence one can ask the following:

Question If $Y \subset X$ is a compact totally geodesic proper submanifold inside a locally symmetric space of noncompact type, when is $Y$ a retract of $X$ ?

Remark (1) In the case where $X$ is a (nonproduct) higher rank locally symmetric space of noncompact type, one cannot find a proper totally geodesic submanifold $Y \subset X$ which is a retract of $X$. Indeed, if there were such a submanifold, then the morphism $\rho: \pi_{1}(X) \rightarrow \pi_{1}(Y)$ induced by the retraction would have to be surjective. By Margulis' normal subgroup theorem [9], this implies that either (1) $\operatorname{ker}(\rho)$ is finite, or (2) the image $\pi_{1}(Y)$ is finite. Since $Y$ is locally symmetric of noncompact type, $\pi_{1}(Y)$ cannot be finite, and hence we must have finite $\operatorname{ker}(\rho)$. But $\operatorname{ker}(\rho)$ is a subgroup of the torsion-free group $\pi_{1}(X)$, hence must be trivial. This forces $\pi_{1}(X) \cong \pi_{1}(Y)$, which contradicts the fact that the cohomological dimension of $\pi_{1}(X)$ is $\operatorname{dim}(X)$, while the cohomological dimension of $\pi_{1}(Y)$ is $\operatorname{dim}(Y)<\operatorname{dim}(X)$. This implies that no such morphism exists, and hence no such submanifold exists. The authors thank C Leininger for pointing out this simple argument.

(2) In the case where $X$ has rank one, the question is more delicate. Some examples of such retracts can be found in a paper by Long and Reid [8]. We remark that in this case, the application to bounded cohomology is not too interesting, as Mineyev [13] has already shown that the comparison map in this situation is surjective in all dimensions $\geq 2$.

Finally, we conclude this paper by pointing out that the Okun maps, while easy to define, are geometrically very complicated. We illustrate this with a brief comment on the singularities of the tangential maps between locally symmetric spaces of noncompact type and their nonnegatively curved dual spaces. More precisely, for a smooth map $f: X \rightarrow X_{u}$, consider the subset $\operatorname{Sing}(f) \subset X_{u}$ consisting of points $p \in X_{u}$ having the property that there exists a point $q \in X$ satisfying $f(q)=p$, and $\operatorname{ker}(d f(q)) \neq 0$ 
(where $d f: T_{q} X \rightarrow T_{p} X_{u}$ is the differential of $f$ at the point $q$ ). We can now ask how complicated the set $\operatorname{Sing}(h) \subset X_{u}$ gets for $h$ a smooth map within the homotopy class of the Okun maps.

Proposition 5.2 Let $X$ be a closed, locally symmetric space of noncompact type, $X_{u}$ the nonnegatively curved dual space, $t: \bar{X} \rightarrow X_{u}$ the Okun map from a suitable finite cover $\bar{X}$ of $X$, and $h$ an arbitrary smooth map in the homotopy class of $t$. Consider an arbitrary embedded compact submanifold $N^{k} \subset X$, having the property that (1) $\left[N^{k}\right] \neq 0 \in H^{k}\left(X_{u} ; \mathbb{Z}\right)$, and (2) $N^{k}$ is simply connected. Then we have that $\operatorname{Sing}(h) \cap N^{k} \neq \varnothing$.

Proof Let $h$ be a smooth map homotopic to $t$, and assume that $\operatorname{Sing}(h) \cap N^{k}=\varnothing$. This implies that $d h$ has full rank at every preimage point of $N^{k} \subset X_{u}$. Choose a connected component $S \subset \bar{X}$ of the set $h^{-1}\left(N^{k}\right)$, and observe that the restriction of $h$ to $S$ provides a local diffeomorphism (and hence a covering map) to $N^{k}$. Since $N^{k}$ is simply connected, $S$ is diffeomorphic to $N^{k}$, and $h$ restricts to a diffeomorphism from $S \subset \bar{X}$ to $N^{k} \subset X_{u}$.

Next we observe that the homology class represented by $[S] \in H_{k}(\bar{X} ; \mathbb{Z})$ is nonzero, since this class has image, under $h$, the homology class $\left[N_{k}\right] \neq 0 \in H_{k}\left(X_{u} ; \mathbb{Z}\right)$. But observe that $S \subset \bar{X}$, being simply connected, supports a cellular decomposition with a single 0 -cell and no 1 -cells. In particular, the submanifold $S$ is homotopic to a point in the aspherical space $\bar{X}$, since one can recursively contract all the cells of dimension $\geq 2$ down to the 0 -cell. This forces $[S]=0 \in H_{k}(\bar{X} ; \mathbb{Z})$, giving us the desired contradiction.

We point out a simple example illustrating this last proposition. If $X^{2 n}$ is complex hyperbolic, then $X_{u}=\mathbb{C} P^{n}$, and one can take for $N^{2}=\mathbb{C} P^{1}$ the canonically embedded complex projective line. Topologically, $N^{2}$ is diffeomorphic to $S^{2}$, hence is simply connected, while homologically we have that $\left[N^{2}\right]$ is the generator for the cohomology group $H^{2}\left(\mathbb{C} P^{n} ; \mathbb{Z}\right) \cong \mathbb{Z}$. Proposition 5.2 now applies, and gives us that any smooth map $h$ in the homotopy class of the Okun map must have singular set intersecting the canonical $\mathbb{C} P^{1}$. A similar example appears in the case of quaternionic hyperbolic manifolds, with the singular sets being forced to intersect the canonical $\mathbb{O} P^{1}$ (diffeomorphic to $S^{4}$ ) inside the dual space $\mathbb{O} P^{n}$.

\section{References}

[1] C S Aravinda, F T Farrell, Exotic structures and quaternionic hyperbolic manifolds, from: "Algebraic groups and arithmetic", Tata Inst. Fund. Res., Mumbai (2004) 507-524 MR2094123 
[2] C Bohr, B Hanke, D Kotschick, Cycles, submanifolds, and structures on normal bundles, Manuscripta Math. 108 (2002) 483-494 MR1923535

[3] P Deligne, D Sullivan, Fibrés vectoriels complexes à groupe structural discret, C. R. Acad. Sci. Paris Sér. A-B 281 (1975) Ai, A1081-A1083 MR0397729

[4] M Gromov, I Piatetski-Shapiro, Nonarithmetic groups in Lobachevsky spaces, Inst. Hautes Études Sci. Publ. Math. (1988) 93-103 MR932135

[5] D Husemoller, Fibre bundles, third edition, Graduate Texts in Mathematics 20, Springer, New York (1994) MR1249482

[6] J-F Lafont, R Roy, A note on characteristic numbers of non-positively curved manifolds, to appear in Expo. Math.

[7] J-F Lafont, B Schmidt, Simplicial volume of closed locally symmetric spaces of noncompact type, Acta Math. 197 (2006) 129-143

[8] D Long, A Reid, Subgroup separability and virtual retractions of groups, to appear in Topology

[9] G A Margulis, Discrete subgroups of semisimple Lie groups, Ergebnisse series 17, Springer, Berlin (1991) MR1090825

[10] Y Matsushima, On Betti numbers of compact, locally sysmmetric Riemannian manifolds, Osaka Math. J. 14 (1962) 1-20 MR0141138

[11] J Milnor, Construction of universal bundles. II, Ann. of Math. (2) 63 (1956) 430-436 MR0077932

[12] J Milnor, Morse theory, Based on lecture notes by M. Spivak and R. Wells. Annals of Mathematics Studies, No. 51, Princeton University Press, Princeton, N.J. (1963) MR0163331

[13] I Mineyev, Straightening and bounded cohomology of hyperbolic groups, Geom. Funct. Anal. 11 (2001) 807-839 MR1866802

[14] M Mitra, Cannon-Thurston maps for trees of hyperbolic metric spaces, J. Differential Geom. 48 (1998) 135-164 MR1622603

[15] B Okun, Nonzero degree tangential maps between dual symmetric spaces, Algebr. Geom. Topol. 1 (2001) 709-718 MR1875614

[16] B Okun, Exotic smooth structures on nonpositively curved symmetric spaces, Algebr. Geom. Topol. 2 (2002) 381-389 MR1917058

[17] R Thom, Quelques propriétés globales des variétés différentiables, Comment. Math. Helv. 28 (1954) 17-86 MR0061823 
Department of Mathematics, The Ohio State University

231 West 18th Avenue, Columbus, OH 43210-1174

Department of Mathematics, University of Chicago

5734 S University Avenue, Chicago, IL 60637

jlafont@math.ohio-state.edu, schmidt@math.uchicago.edu

Received: 10 July 2006

Algebraic 83 Geometric Topology, Volume 6 (2006) 